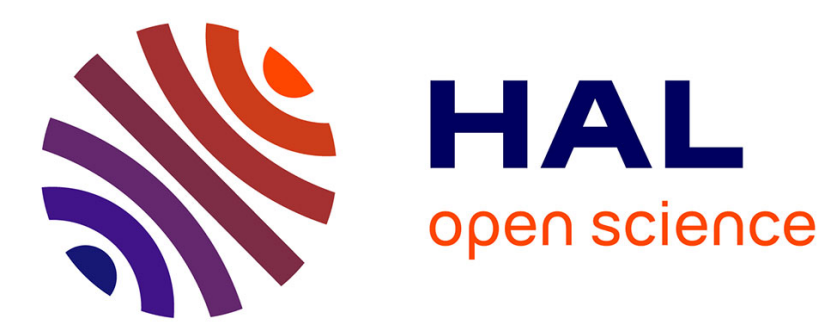

\title{
CONICAL EQUIPOTENTIAL SUBSTRATE FOR LIQUID METAL SOURCES
}

\author{
J. Kubby, B. Siegel
}

\section{To cite this version:}

J. Kubby, B. Siegel. CONICAL EQUIPOTENTIAL SUBSTRATE FOR LIQUID METAL SOURCES. Journal de Physique Colloques, 1986, 47 (C2), pp.C2-107-C2-114. 10.1051/jphyscol:1986216 . jpa00225648

\section{HAL Id: jpa-00225648 https://hal.science/jpa-00225648}

Submitted on 1 Jan 1986

HAL is a multi-disciplinary open access archive for the deposit and dissemination of scientific research documents, whether they are published or not. The documents may come from teaching and research institutions in France or abroad, or from public or private research centers.
L'archive ouverte pluridisciplinaire HAL, est destinée au dépôt et à la diffusion de documents scientifiques de niveau recherche, publiés ou non, émanant des établissements d'enseignement et de recherche français ou étrangers, des laboratoires publics ou privés. 
JOURNAL DE PHYSIQUE

Colloque $\mathrm{C} 2$, supplément au $\mathrm{n}^{\circ} 3$, Tome 47 , mars 1986

page $\mathrm{C} 2-107$

\title{
CONICAL EQUIPOTENTIAL SUBSTRATE FOR LIQUID METAL SOURCES
}

\author{
J.A. KUBBY and B.M. SIEGEL \\ School of Applied and Engineering Physics \\ and \\ The National Research and Resource Facility for Submicron \\ Structures, CornelI University, Ithaca, NY 14853, U.S.A.
}

\begin{abstract}
Morphological changes that occur at an ion bombarded surface as a result of erosion by sputtering can be utilized for the machining of cylindrically symmetric submicron structures. Such structuring has produced tungsten field emitters of conical configuration with variable cone half angle. A conical equipotential surface with an included half angle of $49.3^{\circ}$ would be a useful emitter substrate for experiments designed to produce an equilibrium conical interface to a conducting fluid in an applied electric field. Taylor used a similar structure to support a Taylor cone so that the electric field near the cone could have the proper distribution when, and if, the fluid surface assumed a conical form.
\end{abstract}

\section{I - INTRODUCTION}

Two different techniques have been used in Liquid Metal Ion Sources (LMIS) for localizing the ion emission using the deformation of a liquid metal interface that occurs when the outward stress due to an electric field, $\sigma_{E}=(1 / 8 \pi)(E[V / c m] / 300)^{2}$, on the 1 iquid exceeds the inward stress due to the surface tension, $\sigma_{\gamma}=2 \gamma / R_{0}$ where $\gamma$ is the surface tension and $R_{0}$ the radius of a hemispherical support. The liquid can be lifted into a conical shape along which these stresses are equal with the formation of a sharp apex from which ion emission occurs. The earliest technique involved anchoring of a cusp like filamentary protrusion $/ 1,2 /$ onto the end of a needle shaped appropriately to encourage a single cusp only /3,4/. A later approach employed different boundary conditions $/ 5,6 /$ to encourage the formation and stabilization of a Taylor cone $/ 7 /$. These two techniques are discussed in detail below.

In a High Voltage Electron Microscope (HVEM) study of the liquid metal emission process, Benassayag and Sudraud /8/ found electrohydrodynamic vibrations of the cusp apex in a LMIS. They concluded that these lateral movements, which are not always associated with current fluctuations, could destroy the brightness properties of this source. Further, they felt that better control of liquid metal ion source fabrication and hydrodynamical properties would avoid these instabilities that are incompatible with ion beam technology.

Consideration will be given in this paper as to how the fabrication of the source could be improved. A technique for the generation of conical equipotential emitter substrates on a nanometer length scale is described. Such an emitter substrate would allow for a mode of operation in which the boundary conditions to the potential distribution would encourage the formation and stabilization of a Taylor 
cone that could be fed by an adlayer of neutral material. Operation with this emitter would reduce the volume of fluid in the apex structure to less than 0.001 percent of the volume in a cusp anchored to the end of a $1 \mu \mathrm{m}$ radius of curvature needle operating in the capillary wave mode $/ 1,2,3,4 /$. Operation around the local equilibrium point found by Taylor /7/ should stabilize the configuration which forms at the emitter apex. As this method involves different boundary conditions relative to the standard LMIS, the potential distribution problem solved by Taylor /7/ will be discussed and contrasted to the capillary wave problem that is the basis of the work by Clampitt et al $/ 3,4 /$.

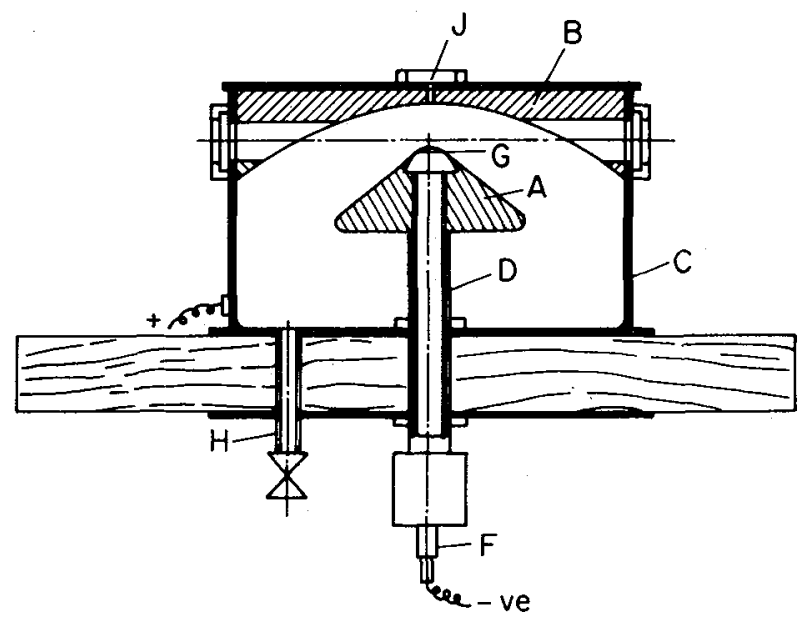

Fig.1 - Taylor's chamber for producing the field necessary for a conical interface to a conducting fluid.

\section{II - TAYLOR'S BOUNDARY CONDITIONS}

In an attempt to find under what conditions a conical liquid point could exist in equilibrium within an applied field, Taylor /7/ designed the experimental apparatus shown in figure 1 to produce such a point under controlled conditions. To balance the macroscopic surface tension which would exist everyhere on a conical surface with the stress on that surface produced by an electric field, Taylor found that the potential would have to be;

$$
V(r, \theta)=V_{0}\left[1-\left(r / r_{0}\right)^{1}{ }^{2} p_{1 / 2}(\cos (\theta))\right]
$$

where $P_{1 / 2}(\cos (\theta))$ is the Legendre function of order $1 / 2, r$ the distance from the apex of the conical equipotential $V(r, \theta)=V_{0}, r_{0}$ the distance from the cone apex to the counter-electrode described below in equation 2, and $\theta$ the angle measured from the axis of the conical equipotential. The cone along which the potential is constant and equal to $V_{0}$ has a semi-vertical angle $\alpha=\pi-\theta_{0}=49.3^{\circ}$, where $\mathrm{P}_{1 / 2}\left(\cos \left(\theta_{0}=130.7\right)\right)=0$. To make it possible to produce the field represented by the potential in equation (1) between two metallic surfaces, Taylor /7/ constructed the chamber shown in figure 1 . Surface $A$ in this figure was a truncated aluminum cone of semi-angle $49.3^{\circ}$. Surface $B$ was an aluminum disk hollowed out so that its lower surface was represented by the equation;

$$
r(\theta)=r_{0} /\left[P_{1} / 2(\cos (\theta))\right]^{2}
$$

The object of this disk was to insure that the electric field near the cone could have the calculated distribution of equation (1) when, and if, the fluid surface assumed a conical form. Cone $A$ was truncated so that its upper edge was a horizontal circle $1 \mathrm{~cm}$ in diameter which could form the lower edge of a conical liquid surface if such a surface could in fact be formed. Using this apparatus Taylor found a point of local equilibrium in the static limit he was considering for the potential distribution surrounding an infinite cone and counter-electrode of the idealized form that he was able to approximate sufficiently with his experimental apparatus. 


\section{III - LIQUID METAL SOURCE GEOMETRY; THE CAPILLARY WAVE PROBLEM}

Clampitt and Jefferies $/ 3,4 /$, in developing miniature ion sources for analytical instruments, saw drawbacks in the mode of operation involving the formation and stabilization of a Taylor cone for the generation of ions and instead developed a needle geometry with a planar aperture as the counter-electrode. The curved surface of the small radius needle was meant to reduce the voltage necessary for the formation of a cusp, and was shaped appropriately to encourage a single cusp only. An array of similar cusps form caused by the impression of an electrostatic

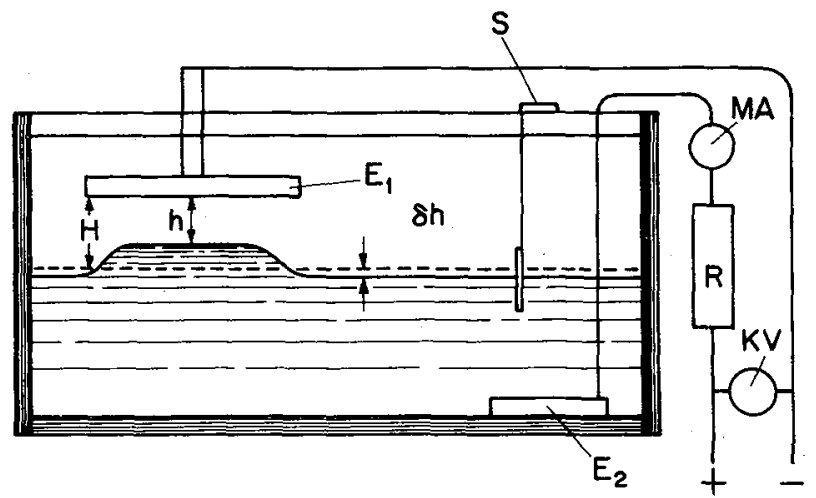

Fig. 2 - Chamber for producing the initialiy uniform vertical electric field above a planar conducting surface in the capillary wave problem.

field on a plane infinite conducting liquid surface /1/. This capillary wave problem was also studied by Taylor /2/ with the experimental apparatus shown in figure 2. Here a local vertical displacement of the interface will concentrate the lines of force and so increase the vertical force on this interface. If this increase is great enough to counter-balance the pressure drop due to gravity and the surface tension, the interface becomes unstable and cusps develop. Note the stricter boundary conditions imposed at both the anode and counter-electrode of figure 1 relative to the anode and counter-electrode system of figure 2 . The motive behind the clampitt et al $/ 3,4 /$ design was a desire to reduce to a minimum the size of the liquid exposed to the high field $/ 9 /$, thereby minimizing the development of instabilities on the ion emitting surface that would lead to the emission of droplets. Presumably they felt that the volume of fluid in a cusp that would form at the apex of an electrochemically polished needle was less than that which would form with an anode similar to that shown in figure 1 and thus avoid droplet formation. This condition would certainly be true for an anode fabricated with macroscopic dimension as was Taylor's $/ 7 /$ or Kingham and Bell's $/ 5,6 /$. However this situation would not obtain for a Taylor type anode fabricated with dimensions on a length scale that is much smaller than the $1 \mu \mathrm{m}$ radius of curvature of the needle anode used by Clampitt.

Experimental studies $/ 4,5,6,8,10,11,12 /$ show that conical shapes which are similar to those found by Taylor $/ 7 /$ form, in situations that are not too far removed from the static limit considered by Taylor, even when the boundary conditions only approximate his experimental set up. These results confirm Taylor's hypothesis of a Tocal equilibrium for the Taylor cone, therefore it would be useful to have an emitter structure that would support and stabilize this cone.

\section{IV - ION BEAM PROCESSING OF LMIS EMITTERS}

By bombarding an electrochemically polished emitter tip along its axis, the variation of the sputter yield efficiency with the angle of incidence between a collimated ion beam and the normal to the local macroscopic surface of the tip can 
be used to contour the apex of the emitter in a predictable manner. Variation in sputter yield with angle of incidence has been described previously /13/ so it will only be discussed briefly here. Essentially, for angles of incidence which are not too far removed from the normal, the sputter efficiency initially increases with. increasing angle of incidence as the mean depth of energy deposition nears the target surface where sputtering occurs; a near $1 / \cos (\theta)$ effect obtains as shown in figure 3(a). However as a characteristic glancing angle is approached, determined by the projectile/target combination as well as the ion's incident energy /14/, the beam is reflected off the target surface decreasing the energy deposition within the target and thus reducing the sputter efficiency to zero at total reflection. This combination of effects leads to the characteristic form for the sputter yield shown in figure $3(\mathrm{~b})$. The orientation between the beam and target surface that is

\section{Figure 3}

(a)

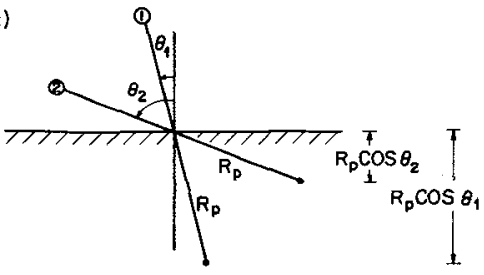

(b)

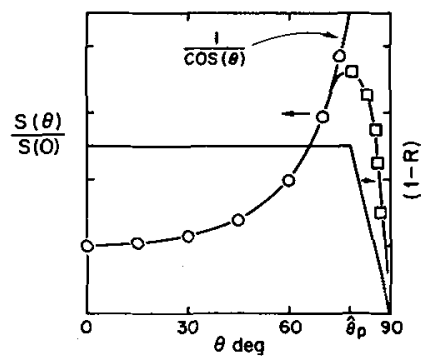

\section{Figure 4}

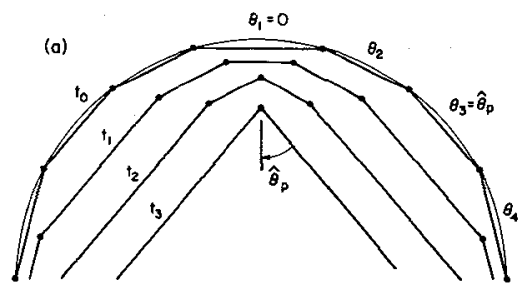

(b)

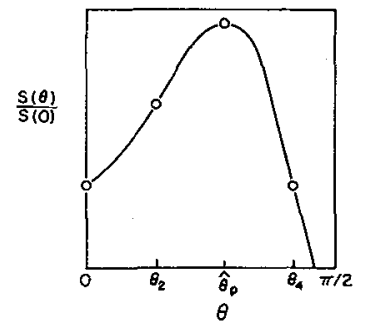

Fig. 3 - For a given projected range $R_{p}$, as the angle of incidence increases away from the normal the energy deposition occurs nearer the surface as shown in (a). In (b) is shown the initial rise of sputter efficiency with increased energy deposition near the surface, and the final reduction beyond $\theta_{p}$ which is to be associated with ion reflection off the target.

Fig. 4 - The hemisphere in (a) is approximated by the straight line segments at the orientations marked on the sputter yield efficiency curve in (b). As erosion proceeds the line segment at $\theta_{p}$, which sputters most efficiently, overtakes all other orientations at time $t_{3}$.

marked $\theta_{p}$ in this figure is the orientation which sputters most efficiently; it is steep enough to bring the energy deposition close to the surface but not so steep as to reflect off all of the beam. A segment of the surface that is at this orientation will recede rapidly along this direction under ion beam erosion. Figure $4(\dot{a})$ shows the morphological development to be expected for an initially hemispherical target bombarded from above. The hemisphere is approximated by the 
four orientations that are marked on the sputter yield curve in figure 4 (b). As erosion proceeds, these surface elements move parallel to themselves at the relative velocities determined by their sputter efficiencies. By the time marked $t_{2}$, the orientation at $\theta_{2}$ has overtaken the slower (lower efficiency for sputtering) orientation at $\theta_{1}$ forming a conical cap of semi-angle $\theta_{2}$. As the erosion proceeds, the element at orientation $\theta_{p}$, which sputters most efficiently, overtakes all other elements to form the equilibrium endform of a cone with half angle $\theta_{p}$ at time $t_{3}$. A similar morphological development is expected for an initial profile that is parabolic for it also includes all surface orientations including that which sputters most efficiently. After electropolishing, a tip is approximately paraboloidal. The conical endform which results after erosion, with cone half angle equal to 49.3 degrees, provides the proper geometry at the anode to satisfy the potential distribution boundary conditions of the Taylor cone problem.

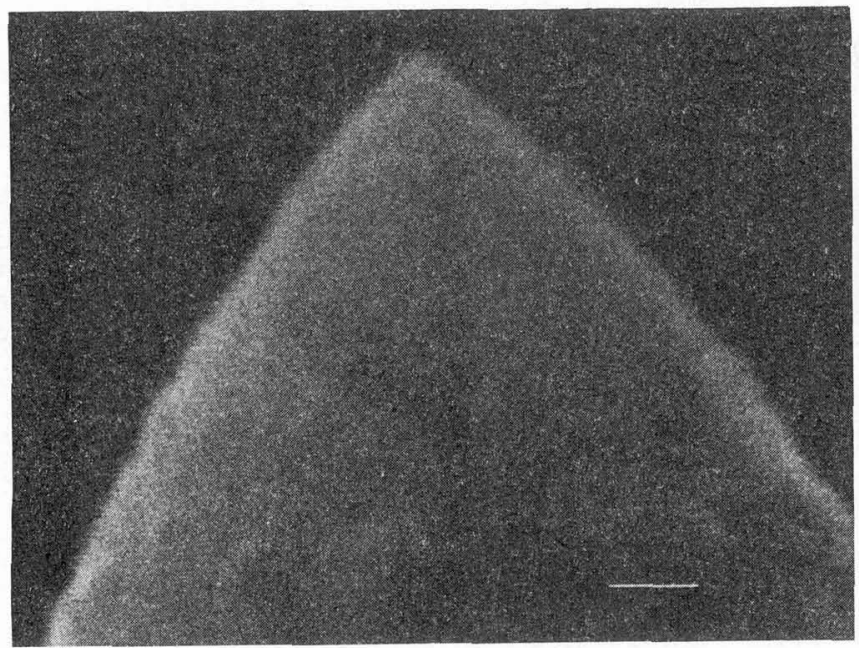

Fig. 5 - SEM

micrograph of the conical equitibrium configuration after a long erosion time. The marker shows a 100 $\mathrm{nm}$ length.

\section{$V$ - EXPERIMENTAL}

A detailed description of the initial emitter preparation as well as the characteristics of the milling apparatus was given elsewhere $/ 13 /$. Oniy an outline will be given here.

The emitter tip was in some cases single crystal $\langle 100\rangle$ oriented tungsten 5 mil four PZR wire, and in other cases drawn polycrystalline tungsten or iridium.

Electropolishing sharpened the tip to an approximate $1 \mu \mathrm{m}$ radius of curvature, similar to the sharpness of a sewing needle.

The ion mill that was used for the erosion experiments, with a hollow anode source and rotating specimen, is similar to the configuration used in ion thinning of TEM specimens. As much as $500 \mu \mathrm{A}$ of $\mathrm{Ar}^{+}$as well as other species of inert and reactive gases in the energy range of 3 to $15 \mathrm{keV}$ were provided by this source. A hollow anode configuration provides a well collimated beam of approximately $1 \mathrm{~mm}$ diameter. Further beam definition is given by an aperture at the front of a target chamber that contains the emitter tip as well as an aperture at the exit of the source. Mechanical rotation of the entire target assembly at an angular velocity $\omega$ of $1 \mathrm{radian} / \mathrm{sec}$ averages the ion flux to give beam heterogeneity and provides a reference axis for morphological development. 
VI - RESULTS

An SEM micrograph, figure 5, shows the equilibrium conical endform obtained for an $\mathrm{Ar}+\mathrm{W}$ projectile/emitter combination. The included half angle is a function of the incident ion's energy as well as the mass ratio $M_{1} / M_{2}$ where $M_{1}$ is the mass of the incident ion and $M_{2}$ is that of the target atoms $/ 14 /$. The transient cone half angle is a function of dose $/ 14 /$. A bar in the lower corner delineates a length of $100 \mathrm{~nm}$. The conical section extends to a base greater than $1 \mu \mathrm{m}$. A series of SEM micrographs in /13/ show the dynamical development of the emitter contour as this equilibrium endform is approached. The computer generated plots in /13/ show the morphological development expected in accordance to the first order erosion theory 115\%. Details of the cone apex are at the resolution limit of the SEM and can be masked by the ubiquitous carbon contamination. In the TEM micrograph included in figure 6 these details can be seen. Here the cone apex is seen to be rounded with a $20 \mathrm{~nm}$ radius of curvature that is comparable to the ion penetration depth of the Art ion within the $W$ target matrix $/ 16,17 /$.

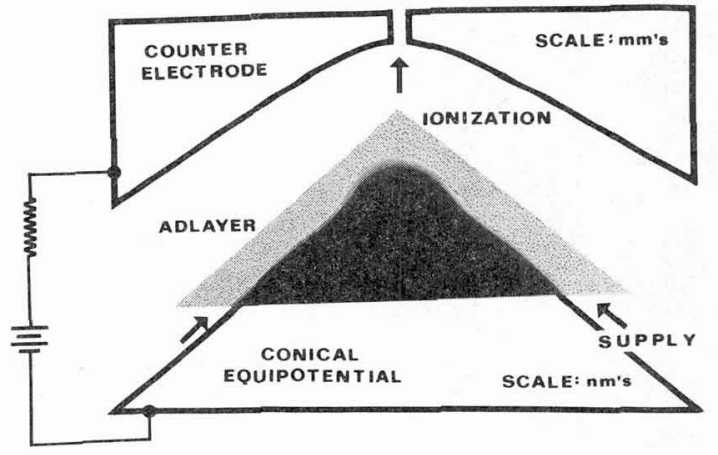

Fig. 6 - Anode and counter-electrode system for producing the field necessary for a conical interface to a conducting fluid in the Liquid Metal Ion Source. The anode is a TEM micrograph of the emitter apex that has a $20 \mathrm{~nm}$ radius of curvature.

\section{VII - DISCUSSION}

Taylor had found that a conducting fluid could exist in static equilibrium in the form of a cone under the action of an electrostatic field, but only when the semi-vertical angle was $49.3^{\circ}$. His apparatus was constructed in such a way that the necessary field could be set up and fluid surfaces that were very nearly conical with the hypothesized half angle of $49.3^{\circ}$ were observed. Other experimental observations of the LMIS emitting structure $/ 4,5,6,8,10,11,12 /$ also show this structure to be a shape of local equilibrium. Therefore it would be useful to have an emitter structure that would support and stabilize this liquid metal cone similar to the apparatus of Taylor $/ 7 /$, but reduced in size. The ion erosion fabrication technique discussed has produced conical emitter equipotential substrates as shown in figures 5 and 6 . Control of the emitter apex in the LMIS has been suggested previously by Yamaguchi et al /18/ where the top curvature of the tip in a needle type Gallium ion source was re-etched to improve current stability, although no details of the process were given.

The combination of anode and counter-electrode is shown in figure 6 . The boundary conditions that are imposed by the electrodes in this figure should be compared to the boundary conditions imposed by the system of electrodes shown in figure 1 . The electrodes in figure 6 are meant to replicate, albeit on a reduced length scale, those in figure 1 . The boundary conditions imposed by the system of electrodes shown in figure 6 should also be contrasted with the boundary conditions imposed by the electrodes of figure 2. The stricter boundary conditions imposed by the set of electrodes in figure 6 are meant to be an improvement on the boundary conditions imposed by the electrodes of figure 2 that are similar to the boundary conditions imposed by the needle geometry of the conventional LMIS $/ 3,4 /$. 
A thin adlayer has been drawn on the emitter in figure 6 which supplies the emission from the Taylor cone formed at the emitter apex. An optimal operating temperature $/ 19 /$ would allow the level of supply to equal the rate of emission and in this way avoid the instabilities associated with large volumes of fluid being exposed to high field /9/. Reduction of the base dimension of the emitter that supports the Taylor cone from the $1 \mu \mathrm{m}$ or larger radius of curvature needle in the Clampitt et al /3,4/ configuration to the $20 \mathrm{~nm}$ radjus of curvature emitter shown in figure 6 would give a factor of $\left(20 * 10^{-9} / 1 * 10^{-6}\right)^{3}$ or $10^{-5}$ reduction. in the volume of fluid exposed to the high field within the Taylor cone.

The volume of fluid exposed to the high field can also affect the energy spread of the LMIS source. With the reduction of fluid exposed to the high field in both the supply layer and the cone structure it is expected that the emission of droplets should be reduced $/ 4,9 /$. These droplets and the non-stationary effects assocjated with the droplet emission process; i.e. transient perturbations of surface geometry and electric field at the emitter tip and the acceleration of atomic ions in this time dependent (non-conservation) electric field can affect the energy spread in the LMIS as discussed by Papadopoulos et al in $/ 20 /$.

Operation about a point of local equilibrium should also help suppress gross movements of the entire liquid protrusion /21/ that should be avoided for "ion optical applications. As the cone, in the fabrication technique that has been described, is aligned to the axis of the needle it should reduce the offset of the angular current distribution from 0 degrees found by Papadopoulos et al $/ 19 /$. Localization of the high field region by the emitter substrate should decrease sensitivity to contamination away from the cone apex. Contamination can cause the position of the cusp to be unstable on the tip configuration of the conventional LMIS as well as provide growth sites for parasitic cusps which can occur even at low voltages $/ 8 /$.

\section{VII - ACKNOWLEDGMENTS}

The authors wish to express their gratitude to the staff of the Materials Science Center; John Hunt, Margaret Craft, and Ray Coles, not only for their service and patient training on the SEM and TEM but also for providing the ion mill, Jane Jorgensen for the drawings, and Marcia Kelley for the photography. We would also like to thank Elizabeth A. Roach and Linda Habetler of the Clark Hall Physical Sciences Library for assistance in literature searches and diversion. We appreciated the help from members of the Department of Applied Physics; the typing done by Bonni Jo Davis, the guidance of Dr. Gary R. Hanson, and Paul R. Schwoebel for interesting and informative discussions.

This work was supported by DARPA under ONR contract N00014-80-C-0587.

\section{REFERENCES}

/1/ J. Melcher, Phys. Fluids 4, (1961) 1348.

$12 /$ G.I. Taylor and A. McEwan, J. Fluid Mech. 22, (1965) 1.

/3/ R. Clampitt, K.L. Aitken, and D.K. Jefferies, J. Vac. Sci. Technol. 12, (1975) 1208 .

/4/ R. Clampitt and D.K. Jefferies, Nucl. Instr. and Methods 149, (1978) 739.

15/ D.R. Kingham and A.E. Be11, Journal De Physique C9, (1984) 139 .

$16 /$ D.R. Kingham and A.E. Be11, App 1. Phys. A36, (1985) 67.

17/ Sir Geoffrey Taylor F.R.S., Proc. Ray. Soc. London A 280, (1964) 383.

18/ G. Benassayag and P. Sudraud, UTtramicroscopy 16, (1985) 1.

$19 /$ K.L. Aitken and G.L.R. Mair, J. Phys. D13, (1980) 2165.

/10/ A. Wagner, T. Venkatesan, P.M. Petroff, and D. Barr, J. Vac. Sci. Technol. 19 , (1981) 1186.

/11/ F. Sudraud, C. Colliex, and J. Van De Walle, Le Journal De Physique Letters 40 , (1979) L207.

/12/ G. Benassayag and P. Sudraud, Journal De Physique C9, (1984) 223.

113/ Joel A. Kubby and Benjamin M. Siegel, Proc. 29th Int. Symp. on Electron, Ion and Photon Beams, to be published in J. Vac. Sci. Technol., Jan. 1986. 
/14/ M.J. Witcomb, J. Mat. Sci. 9, (1974) 1227.

/15/ George Carter, Boris Navinsek, and James L. Whitton, in Sputtering by Particle Bombardment II, edited by R. Behrisch (Springer-Verlag, Berlin 1983) pg. 257.

/16/ Joel A. Kubby and Benjamin M. Siegel, Proc. 11th Inter. Conf. on Atomic Collisions in Solids, to be published in Nucl. Instr. and Methods.

117/ Joel A. Kubby and Benjamin M. Siegel, to be published in the Proceedings of the Erosion and Growth of Solids Stimulated by Atom and Ion Beams, NATO Advanced Study Institute, Heraklion, Crete, Greece (1985).

/18/ H. Yamaguchi, A. Shimase, S. Haraichi, and T. Miyauchi, J. Vac. Sci. Technol. B3, (1985) 71.

/19/ Gary Hanson, SRI Microelectronics Series Vol. II, (1984) 29.

$120 /$ S. Papadopoulos, D. Barr, W.L. Brown, and A. Wagner, Journal De Physique C9, (1984) 217.

/21/ P.D. Prewett and D.K. Jefferies, J. Phys. D13, (1980) 1747. 